
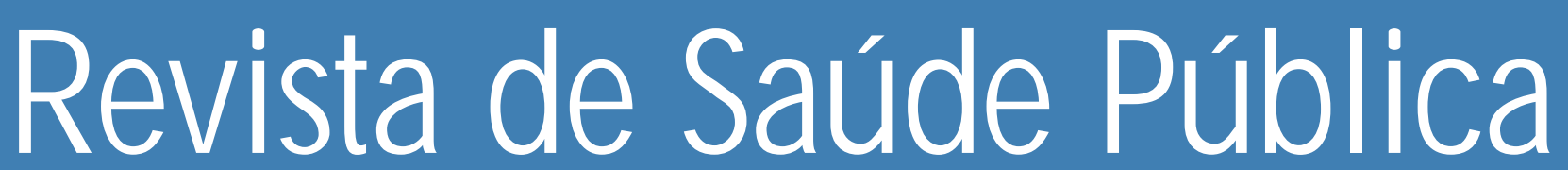

\begin{tabular}{llllllll}
\hline & 0 & $U$ & $R$ & $N$ & $A$ & $L$
\end{tabular}

$0 F$

P U B L I C

H E A L T H

\title{
Evaluación del estado de salud bucodental en preescolares: estudio epidemiológico longitudinal (1993-1994), Córdoba, Argentina*
} 0 ral health condition evaluation of kindergarten children: longitudinal epidemiologic study (1993-1994), Córdoba, Argentina

Luis José Battellino, Lila Susana Cornejo, Susana Tereza Dorronsoro de Cattoni, Elba Rosa Luna Maldonado de Yankilevich, Silvia Edith Calamari, Ana Isabel Azcura y Carolina Virga Cátedra de Q uímica y Física Biológicas de la Facultad de O dontología de la U niversidad Nacional de Córdoba (FO/U NC) Córdoba, Argentina (L. J. B., A. I. A.); Cátedra de Biología de la FO/U NC (L. S. C.); Cátedra de Introducción a la Q uímica y Física Biológicas de la FO/U NC (S. T. D. C., S. E. C.); Cátedra de Introducción a la O dontología de la FO/U NC (E. R. L. M. Y.); Cátedra de Farmacología y Terapéutica de la FO/UN C (C. V.) 


\title{
Evaluación del estado de salud bucodental en preescolares: estudio epidemiológico longitudinal (1993-1994), Córdoba, Argentina*
}

\section{O ral health condition evaluation of kindergarten children: longitudinal epidemiologic study (1993-1994), Córdoba, Argentina}

\begin{abstract}
Luis José Battellino, Lila Susana Cornejo, Susana Tereza Dorronsoro de Cattoni, Elba Rosa Luna Maldonado de Yankilevich, Silvia Edith Calamari, Ana Isabel Azcura y Carolina Virga

Cátedra de $\mathrm{Q}$ uímica y Física Biológicas de la Facultad de $\mathrm{O}$ dontología de la U niversidad $\mathrm{N}$ acional de Córdoba (FO/U NC) Córdoba, Argentina (L. J. B., A. I. A.); Cátedra de Biología de la FO /UNC (L. S. C.); Cátedra de Introducción a la Q uímica y Física Biológicas de la FO /U N C (S. T. D. C., S. E. C.); Cátedra de Introducción a la O dontología de la FO/U NC (E. R. L. M. Y.); Cátedra de Farmacología y Terapéutica de la FO/UNC (C. V.)
\end{abstract}

\begin{abstract}
Resumen
Se realizó un estudio longitudinal de un año de duración en una muestra ( $\mathrm{n}=$ 820) de la población preescolar de 4 años de la Ciudad de Córdoba, Argentina, para establecer la participación de diversas variables en la incidencia de caries. Los índices ceo-d, ceo-s, de higiene oral y de salud bucodental, como también las tasas de incidencia y los riesgos relativos de caries estuvieron inversamente relacionados con el Nivel Económicosocial (NES) de los niños. En los preescolares del NES III (proletariado típico, proletariado no típico y subproletariado), el riesgo relativo de experimentar caries fue casi cinco veces mayor $(\mathrm{RR}=4,9)$ que en el NES I (burguesías empresarial y gerencial). En el NES I, la mayoría de las nuevas lesiones se localizaron en las superficies lisas $(61,2 \%)$, mientras que en el NES III afectaron predominantemente las superficies oclusales de los molares $(66,3 \%)$. El consumo diario de azúcares fue mayor en los niños del NES III, pero la experiencia de caries se correlacionó débilmente con la cantidad o frecuencia de ingesta de estos carbohidratos $(r=0,40$ y 0,52, respectivamente). No se registraron diferencias significativas interniveles en los parámetros bioquímicos salivales analizados. El cepillado dental asistido y las aplicaciones tópicas fluoruradas disminuyeron fuertemente la incidencia de caries en los niños del NES III, reduciendo las correspondientes tasas a valores muy próximos a los del NES I $(0,31,0,23$ y 0,22 vs. 0,21$)$. Se concluye que los niños del NES III, por su susceptibilidad a la caries, deberían ser asistidos precozmente con medidas preventivas eficaces, como lo son el cepillado dental asistido y las aplicaciones tópicas fluoruradas.
\end{abstract}

Encuestas de salud bucal. Caries dental, epidemiología. Índice CPO.

\footnotetext{
* Parte do trabalho foi subsidiada pelo "Consejo de Investigaciones Científicas y Tecnológicas de la Provincia de Córdoba" e pela "Secretaría de Ciencia y Tecnología de la Universidad Nacional de Córdoba, Argentina".

Correspondência para/Correspondence to: Luis José Battellino - Facultad de Odontología de la Universidad Nacional de Córdoba, Pabellón Argentina - Ciudad Universitaria (Agencia 4) 5000 Córdoba, Argentina. E-mail: luisjosé@ odo1.odo.unc.edu.ar

Recebido em 19.3.1996. Reapresentado em 31.10.1996. Aprovado em 20.11.1996.
} 


\begin{abstract}
A one-year longitudinal survey was carried out on a sample of the Cordoba City 4-year old kindergarten population $(n=820)$; so as to determine the role of several variables upon the incidence of caries. The dmf-t, dmf-s, oral hygiene and oral health indexes as well as incidence rates and caries relative risks of caries were inversely related to the socioeconomic level (SEL) of the children involved. Thus in the SEL III (typical proletariat, non-typical proletariat and sub-proletariat) children, the relative risk of caries was almost five times higher $(R R=4.9)$ than in the SEL I (entrepreneureal and managerial bourgeoisie) childreen. In SEL I, almost all new lesions occurred on smooth surfaces (61.2\%), while in SEL III the molar occlusal faces were mainly affected $(66.3 \%)$. Daily sugar intake was higher in SEL III children but experience of caries showed poor correlation to the amount $(r=0.40)$ and frequency $(r=0.52)$ of carbohydrate intake. No significant interlevel differences were observed in the biochemical salivary parameters analyzed. Assisted toothbrushing and fluoride topications strongly lowered the incidence of caries among SEL III children, also making the corresponding rates fall almost to SEL I values $(0.31,0.23$ and 0.22 vs. 0.21). In conclusion, SEL III children should be trated prophylactically with effective preventive measures, because of their susceptibility to caries. Such preventive measures include assisted toothbrushing and fluoride topications.
\end{abstract}

Dental health surveys. Dental caries, epidemiology. DMF index.

\section{INTRODUCCIÓN}

La prevalencia de caries dental ha experimentado una fuerte reducción en el curso de las dos últimas décadas en la mayoría de los países industrializados y en algunos en vías de desarrollo ${ }^{16,24,26}$. El mayor impacto en este descenso es atribuible al uso extensivo de fluoruros ${ }^{16,26}$. Sin embargo, muchos países $^{4}$ y/o grupos sociales ${ }^{40}$ con menores recursos económicos parecen no haberse beneficiado mayormente de tales estrategias preventivas.

Al comparar los resultados de recientes estudios de corte transversal ${ }^{7,47}$ en la población preescolar de 5 años de la Ciudad de Córdoba (Argentina) con los datos obtenidos por Battellino y cols. ${ }^{1}$, en una investigación similar llevada a cabo en 1974, se deduce que en el intervalo de los últimos veinte años se ha producido un aumento $(26,7 \%)$ en la proporción de niños sin experiencia de caries y una significativa reducción $(55,9 \%)$ en el índice ceo-d. En ambos estudios epidemiológicos pudo comprobarse asimismo que el estado de salud bucodental seguía un gradiente social, donde la frecuencia e intensidad de caries y las necesidades de tratamiento restaurativo eran mayores conforme descendía la condición socioeconómica de los niños. Tales diferenciales no pudieron explicarse en base a hábitos alimentarios y de higiene bucal, utilización de servicios de atención odontológica y niveles salivales de algunos parámetros bioquímicos e inmunológicos.
Por cuanto la caries dental es una enfermedad de avance lento, el diseño utilizado para la investigación no permitía establecer si la magnitud de dichas variables en la instancia de realizar el examen clínico eran equivalentes a las que existían cuando comenzó la enfermedad dental. En razón de ello, en 1993 se inició un estudio prospectivo en la población infantil de 4 años de la Ciudad de Córdoba, cuyo objetivo principal era determinar el grado de participación que tenían diversos factores de riesgo en la producción de caries en preescolares de diferente condición socioeconómica. En el presente trabajo se exponen y analizan los resultados de esa investigación.

\section{MATERIAL Y METODO}

Como la metodología utilizada en la investigación ha sido previamente descripta ${ }^{7,47}$, se expondrá solamente un resumen de la misma.

Población y muestra. En base a la información proporcionada por el Ministerio de Educación de la Provincia de Córdoba, se seleccionaron los Jardines de Infantes oficiales y privados de la Ciudad de Córdoba que contaban con divisiones de prejardín (4 años) y nivel inicial (5 años). Utilizando el procedimiento aleatorio, de cada uno de dichos jardines se escogieron unidades hasta constituir una muestra de 850 niños.

Categorización socioeconómica. Mediante encuesta por cuestionario que completaron los padres se obtuvo información respecto a la condición laboral y nivel de escolaridad del principal responsable del sustento familiar, a partir de la cual los niños de la muestra fueron agrupados en tres 
categorías socioeconómicas ${ }^{5,21}$, a saber: Primer Nivel Económicosocial (NES I = burguesía empresarial y gerencial), Segundo Nivel Económicosocial (NES II = pequeña burguesía tradicional y nueva pequeña burguesía) y Tercer Nivel Económicosocial (NES III = proletariado típico, proletariado no típico y subproletariado).

Diseño metodológico. Se realizó un estudio longitudinal en el período setiembre-noviembre de 1993 y setiembre-noviembre de 1994. Comenzaron el estudio un total de 820 niños (cohorte principal), pero debido al desgranamiento lo completaron sólo 720 (87,8\%). El tamaño, edad promedio y distribución por sexo en los tres NES fueron casi idénticos.

Examen clínico-odontológico. Se llevó a cabo en los Jardines de Infantes durante el horario de actividades escolares, en un local adecuadamente iluminado con luz natural, utilizando instrumental exploratorio de rutina. El mismo operador (ERLM de Y) realizó todos los exámenes odontólogicos, el cual consistió en el recuento y clasificación del total de dientes presentes en boca, detección de elementos y superficies cariados, con extracción indicada y obturados, presencia de anomalías dentarias, alteraciones de la oclusión, grado de inflamación de las encías y estado de la higiene bucal.

Los criterios utilizados para la determinación de caries fueron los propuestos por la $\mathrm{OMS}^{46}$, sin que se realizaran exámenes radiológicos. En base al recuento de dientes con caries activa, extracción indicada y obturaciones se construyeron los índices ceo-d y ceo-s ${ }^{14}$. La tasa de incidencia porcentual (TI\%) para el período '93-'94 fue calculada a partir de la siguiente relación:

$$
\mathrm{TI} \%=\frac{\sum \mathrm{x}_{\mathrm{i}} / \mathrm{n}_{\mathrm{i}}}{\mathrm{n}} \cdot 100
$$

donde $x_{\mathrm{i}}$ es el número de nuevas superficies dentarias cariadas durante el período de estudio en el niño $i, n_{\mathrm{i}}$ es el número de superficies dentarias sanas del niño $i$ al comenzar el estudio, $n$ es el tamaño de la muestra en cada nivel económicosocial y 100 es el factor de amplificación. La higiene oral fue determinada mediante la técnica de Greene y Vermillion ${ }^{13}$. El índice de salud bucodental (ISB) fue deducido empleando los criterios establecidos por Koch e col. ${ }^{18}$, correspondiendo el puntaje 10 al estado de boca sana.

Tratamiento preventivo. Independientemente de la cohorte principal, se formaron tres cohortes menores (cohortes de tratamiento), con niños del NES III (en cada una $n=50$ ) que no tenían experiencia de caries, para evaluar los efectos del cepillado dental asistido y de una o dos aplicaciones de geles fluorados, respectivamente. Los geles consistieron en AFP 1,23\% ( $\mathrm{pH} 3,1)$, aplicados durante 4 minutos mediante cubetas. El tratamiento con los geles fluorados se realizó después que un odontólogo (CV) efectuara exhaustiva limpieza mecánica de los dientes, al comenzar el ensayo (una aplicación) y a los seis meses siguientes (dos aplicaciones).

Consumo de azúcares. La información sobre hábitos dietéticos de los niños de la cohorte principal fue obtenida por medio de entrevista personal efectuada a la madre o tutor, en base al recuerdo de las últimas $24 \mathrm{~h}$. Para reducir el impacto que pudieran producir los días de alimentación atípica, se realizaron tres entrevistas (al comienzo, en la mitad y al finalizar el período de estudio), promediando los datos obtenidos ${ }^{37}$. En el interrogatorio se indagó sobre el consumo (cantidad y frecuencia) de los 20 alimentos más habituales en la alimentación infantil a nivel regional. En base a la cantidad de alimento ingerido y de acuerdo a la técnica propuesta por Rugg-Gunn ${ }^{38}$ se calculó la masa de azúcares (glucosa, fructosa, galactosa, sacarosa, lactosa y maltosa) contenidos en dichos alimentos.

Recolección de saliva. La muestra de saliva completa se obtuvo sin estimulación externa, alrededor de 2 horas después de la última ingesta de alimentos, por salivación directa durante 10 minutos en un tubo graduado de polietileno mantenido a $0^{\circ} \mathrm{C}$. A partir de allí, se calculó el flujo salival (mL/minuto). De la muestra total de saliva se separó una pequeña porción destinada al dosaje de Ig A secretoria, reservando el resto para las demás determinaciones. La fracción destinada al examen inmunológico fue calentada a $56^{\circ} \mathrm{C}$ durante $30 \mathrm{~min}$ para inactivar el complemento, luego de lo cual se la sometió a centrifugación a 12.000 x g por 5 min. El sobrenadante límpido se congeló a $-20^{\circ} \mathrm{C}$ hasta el dosaje de Ig A secretoria, el cual fue realizado antes de transcurrida una semana de la recolección. La fracción mayor de saliva fue centrifugada a $12.000 \mathrm{x}$ g durante 10 minutos; los sobrenadantes límpidos se procesaron de inmediato para la cuantificación de sialoperoxidasa, y el resto se conservó no más de 15 días a $-20^{\circ} \mathrm{C}$.

Exámenes bioquímicos e inmunológico. Las determinaciones cuantitativas de calcio, fosfatos, tiocianatos, proteínas totales e Ig A secretoria se realizaron empleando las técnicas propuestas por Ray Sarkar y Chauhan ${ }^{35}$, Chen y col. ${ }^{8}$, Bestt y Dounton ${ }^{2}$, Lowry y col. ${ }^{22}$ y Mancini y col. ${ }^{23}$, respectivamente. Para medir la actividad de sialoperoxidasa se utilizó el procedimiento descrito por Nakano y Asada $^{29}$. Una unidad de sialoperoxidasa correspondió a la actividad de enzima capaz de transformar un $\mu$ mol de sustrato en la unidad de tiempo, bajo las condiciones de ensayo utilizadas.

Evaluación estadística. Los resultados fueron evaluados estadísticamente mediante la prueba del "chi cuadrado" (variables cualitativas) y el análisis de varianza (variables cuantitativas). El límite de significación estadística para establecer diferencias se fijó en el valor $\mathrm{P}=0,05$. Las relaciones entre variables fue estimada mediante un modelo de regresión lineal simple, utilizando el programa computarizado SPSS $^{32}$.

\section{RESU LTAD O S}

Como muestra la Tabla 1, la cantidad promedio de dientes temporarios presentes en boca al comienzo y final del estudio en los niños de la cohorte principal, al igual que el número de piezas permanentes al finalizar el mismo, fueron casi idénticas en los tres niveles económicosociales. En cambio, los índices de caries (ceo-d y ceo-s), las tasas de incidencia, los 
Tabla 1 - Indicadores de salud bucodental en los niños de la cohorte principal al comienzo y final del período de estudio, según su condición socioeconómica. Córdoba, Argentina, 1993-1994.

Table 1 - O ral health indexes in children of the main cohort at the beginning and end of the study period by socioeconomic status. Cordoba, Argentina, 1993-94.

\begin{tabular}{|c|c|c|c|}
\hline & \multicolumn{3}{|c|}{ Nivel Económicosocial } \\
\hline & 1 & II & III \\
\hline Dientes temporarios año 93 & $19,9 \square$ & 20,0 & 19,9 \\
\hline Dientes temporarios año 94 & 19,1 & 19,3 & 19,2 \\
\hline Dientes permanentes año 94 & 1,3 & 1,3 & 1,5 \\
\hline ceo-d año 93 & 1,15 & $1,93 *$ & $3,03 * *$ \\
\hline ceo-d año 94 & 1,61 & $2,79 *$ & $4,23 * *$ \\
\hline Incremento del ceo-d & 0,46 & $0,86 *$ & $1,20 * *$ \\
\hline ceo-s año 93 & 1,51 & $2,64 *$ & $5,17 * *$ \\
\hline ceo-s año 94 & 2,17 & $4,14 *$ & $8,73 * *$ \\
\hline Incremento del ceo-s & 0,66 & $1,50 *$ & $3,56 * *$ \\
\hline N uevas superficies cariadas (c) & 0,35 & $0,71 *$ & $1,72 * *$ \\
\hline Nuevas superficies a extraer (e) & 0,05 & $0,35 *$ & $1,00 * *$ \\
\hline Nuevas superficies obturadas (0) & 0,26 & $0,44 *$ & $0,84 * *$ \\
\hline Tasa de incidencia de caries (TI\%) $\div$ & 0,76 & $1,59 *$ & $3,74 * *$ \\
\hline \multirow[t]{2}{*}{ Riesgo relativo de caries (RR) } & 1,0 & $2,1 *$ & $4,9 * *$ \\
\hline & & $(1,5-3,8)$ & $(2,6-7,2)$ \\
\hline Índice de higiene oral año 93 & 0,02 & 0,03 & $0,08 *$ \\
\hline Índice de higiene oral año 94 & 0,07 & $0,13 *$ & $0,26 * *$ \\
\hline Incremento del índice de higiene oral & 0,05 & $0,10 *$ & $0,18 * *$ \\
\hline Índice de salud bucodental año 93 & 7,4 & $6,1 *$ & $4,7 * *$ \\
\hline Índice de salud bucodental año 94 & 6,9 & $5,3 *$ & $3,5 * *$ \\
\hline Disminución del índice de salud bucodental & 0,5 & $0,8 *$ & $1,2 * *$ \\
\hline
\end{tabular}

- A los efectos de no complicar la exposición de los resultados, en esta tabla y en las siguientes se omiten las correspondientes desviaciones estándar.

* Expresa diferencias estadísticamente significativas respecto al NESI $(\mathrm{P}<0,01)$.

** Expresa diferencias estadísticamente significativas respecto al NES II $(P<0,01)$

$\because$ Indica porcentaje del total de elementos o superficies dentarias en los que ocurrieron las nuevas lesiones de caries.

C $95 \%$.

riesgos relativos y los correspondientes incrementos '93-'94 aumentaron significativamente conforme descendía la categoría socieconómica de los niños. En los tres NES, alrededor de las dos terceras partes del incremento de ceo-s correspondió a la suma de superficies con caries activa (c-s) y con extracción indicada (e-s).
Adoptando arbitrariamente como punto de corte para estimar la susceptibilidad de contraer caries el valor $\mathrm{TI} \%=2$, la proporción de niños que satisfizo esta condición fue del 19,2\% en el NES I, del 35,2\% en el NES II y del 62,0\% en el NES III ( $\mathrm{P}<0,01)$. Esta distribución es coincidente con la que se obtiene tomando como punto de corte un ceo-s mayor o igual

Tabla 2 - Proporción de niños de la cohorte principal con experiencia de caries al comienzo y/o final del período de estudio, según su condición socioeconómica. Córdoba, Argentina, 1993-1994.

Table 2 - Proportion of children of the main cohort with caries experience at the beginning and/or end of the study period, by socioeconomic status. Cordoba, Argentina, 1993-1994.

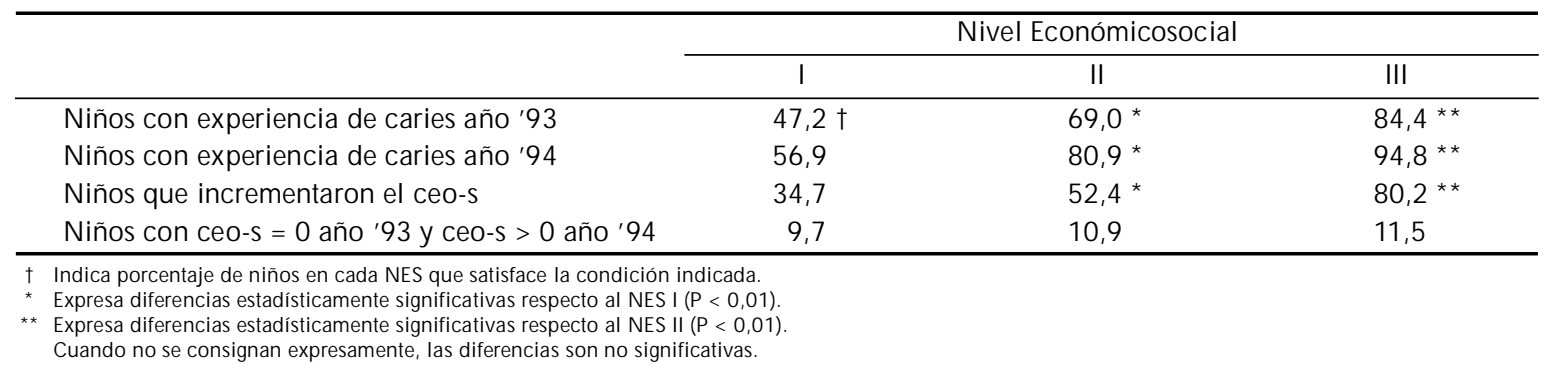


a 5 para el año '94. En los preescolares del NES III, el riesgo relativo de padecer caries resultó aproximadamente 5 veces más alto que en los niños del NES I. Del mismo modo, la proporción de niños con experiencia de caries al comienzo y término del período de estudio, o que incrementaron su índice ceos entre '93 y '94 fue más alta en el NES III, pero no existieron diferencias interniveles en el porcentaje de preescolares que comenzando el estudio con boca sana tuvieron experiencia de caries al completarlo (Tabla 2).

En los tres NES, las nuevas lesiones de caries ocurrieron predominantemente en el primero y segundo molar, especialmente en este último, con menor incidencia en incisivos y caninos (Tabla 3 ). No hubo diferencias significativas interniveles en la distribución de las nuevas lesiones cariosas de acuerdo a los elementos dentarios afectados. En cambio, las lesiones producidas durante el año de estudio se distribuyeron de modo distinto según el NES de pertenencia de los niños: en el NES I se localizaron preferentemente en las superficies lisas, en tanto que en los del NES III estuvieron situadas en su mayoría en las superficies oclusales; en los niños del NES II ambos tipos de superficies resultaron igualmente afectadas.
Tabla 3 - Localización de las nuevas lesiones de caries en elementos y superficies dentarias producidas durante el período de estudio en los niños de la cohorte principal, según su condición socioeconómica. Córdoba, Argentina, 1993-1994.

Table 3 - N ew caries localization on teeth and dental surfaces occurring during the study period in main cohort children, by socioeconomic status. Cordoba, Argentina, 1993-1994.

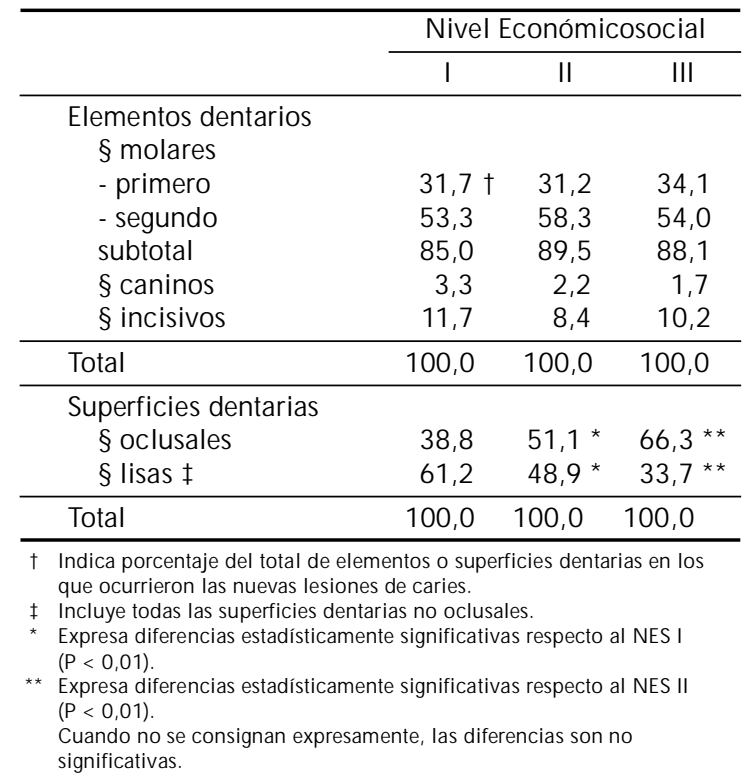

Tabla 4 - Efecto de los tratamientos preventivos al cabo del año de estudio sobre los índices de caries, según condición socioeconómica de los niños. Córdoba, Argentina, 1993-1994.

Table 4 - Effect of preventive treatment of children on caries indexes after one year study, by socioeconomic status. Cordoba, Argentina, 1993-1994.

\begin{tabular}{|c|c|c|c|}
\hline & \multicolumn{3}{|c|}{ Nivel Económicosocial } \\
\hline & I & II & II \\
\hline \multicolumn{4}{|c|}{ § Toda la muestra, sin tratamiento preventivo } \\
\hline ceo-d año's4 & 1,61 & $2,79 *$ & $4,23 * *$ \\
\hline ceo-s año '94 & 2,17 & $4,14 *$ & $8,73 * *$ \\
\hline Tasa de incidencia $\dagger$ & 0,76 & $1,59 *$ & $3,74 * *$ \\
\hline \multicolumn{4}{|c|}{$\S \mathrm{Niños}$ con ceo-s'93 $=0$, sin tratamiento preventivo } \\
\hline ceo-d año '94 & 0,20 & $0,87 *$ & $1,77^{* *}$ \\
\hline ceo-s año '94 & 0,21 & $0,89 *$ & $2,07 * *$ \\
\hline Tasa de incidencia & 0,21 & $0,92 *$ & $2,08 * *$ \\
\hline \multicolumn{4}{|c|}{$\S \mathrm{N}$ iños con ceo-s '93 $=0$, con cepillado asistido } \\
\hline ceo-d año '94 & - & - & 0,31 \\
\hline ceo-s año '94 & - & - & 0,31 \\
\hline Tasa de incidencia & - & - & 0,31 \\
\hline \multicolumn{4}{|c|}{$\S \mathrm{N}$ iños con ceo-s '93 = 0, con una aplicacione tópica fluorurada } \\
\hline ceo-d año '94 & - & - & 0,23 \\
\hline ceo-s año '94 & - & - & 0,23 \\
\hline Tasa de incidencia & - & - & 0,23 \\
\hline \multicolumn{4}{|c|}{$\S N$ iños con ceo-s' $93=0$, con dos aplicaciones tópicas fluoruradas } \\
\hline ceo-d año '94 & - & - & 0,21 \\
\hline ceo-s año '94 & - & - & 0,22 \\
\hline Tasa de incidencia & - & - & 0,22 \\
\hline
\end{tabular}

$\dagger$ Representa el porcentaje de superficies que resultaron cariadas entre los años '93 y ' 94.

* Expresa diferencias estadísticamente significativas respecto al NESI $(P<0,01)$.

** Expresa diferencias estadísticamente significativas respecto al NES II (P $<0,01)$.

Cuando no se consignan expresamente, Ias diferencias son no significativas. 
De acuerdo a lo que señala la Tabla 4 , los índices de caries y la tasa de incidencia de los niños del NES III de las cohortes de tratamiento y que recibieron cepillado dental asistido ó 1-2 aplicaciones tópicas con geles fluorurados, descendieron a valores casi idénticos a los de sus similares no tratados del NES I. Tanto en los niños que practicaron limpieza mecánica de sus dientes bajo supervisión profesional como en los que recibieron tratamiento local con $\mathrm{F}^{-}$, las escasas lesiones de caries se produjeron en las superficies oclusales del primero y segundo molar.

La Tabla 5 muestra que la cantidad diaria promedio de azúcares ingeridos no difiere sustancialmente en los preescolares de los tres NES, pero son significativamente diferentes las proporciones de niños que tuvieron un consumo mayor o igual que el percentilo $70(\mathrm{P}-70 \geq 194,5 \mathrm{~g})$ y menor o igual al percentilo 30 ( $\mathrm{P}-30 \leq 138,9 \mathrm{~g})$ de toda la población estudiada. Por su parte, la frecuencia de consumo de azúcares y las proporciones mayores o iguales al percentilo $70(\mathrm{P}-70 \geq 9,0$ veces $)$ y menores o iguales al percentilo 30 ( $\mathrm{P}-30 \leq 6,4$ veces) fueron significativamente diferentes en los tres NES. Sin embargo, tanto la cantidad como la frecuencia de consumo de azúcares estuvo débilmente correlacionada con la experiencia de caries. Así, el coeficiente $r$ fue de 0,40 para la relación entre la TI\% y la cantidad diaria de azúcar $\left(\mathrm{R}^{2}=0,16\right)$ y de 0,52 para la asociación TI\% y la frecuencia de consumo de azúcar $\left(\mathrm{R}^{2}=0,27\right)$.

Tabla 5- Consumo diario de azúcares (cantidad y frecuencia) en los niños de la cohorte principal, según su condición socioeconómica. Córdoba, Argentina, 1993-1994.

Table 5 - Daily sugar intake (amount and frequency) in main cohort children, by socio-economic status. Cordoba, Argentina, 1993-1994.

\begin{tabular}{|c|c|c|c|c|}
\hline & \multicolumn{3}{|c|}{ Nivel Económicosocial } \\
\hline & & I & II & III \\
\hline \multicolumn{5}{|c|}{ Cantidad } \\
\hline & Gramos por día & 148,2 & 163,8 & 176,0 \\
\hline & Consumo $\geq \mathrm{P}-70 \dagger$ & 20,0 & $31,3 *$ & $39,9 * *$ \\
\hline & Consumo $\leq \mathrm{P}-30 \dagger$ & 52,0 & $39,1 *$ & $27,3 * *$ \\
\hline \multicolumn{5}{|c|}{ Frecuencia } \\
\hline & Veces por día & 5,9 & $7,8 *$ & $9,3 * *$ \\
\hline & Frecuencia $\geq \mathrm{P}-70 \dagger$ & 16,0 & $27,8 *$ & $39,8 * *$ \\
\hline & Frecuencia $\leq \mathrm{P}-30 \dagger$ & 72,0 & $55,1 *$ & $38,1 * *$ \\
\hline \multicolumn{5}{|c|}{$\begin{array}{l}\text { Representa el porcentaje de niños de cada NES que satisface la condición } \\
\text { indicada respecto al total de la población preescolar. } \\
\text { Expresa diferencias estadísticamente significativas respecto al NESI }(\mathrm{P}< \\
0,01) \text {. } \\
\text { *xpresa diferencias estadísticamente significativas respecto al NES II }(\mathrm{P}< \\
0,01) \text {. } \\
\text { Cuando no se consignan expresamente, las diferencias son no } \\
\text { significativas. }\end{array}$} \\
\hline
\end{tabular}

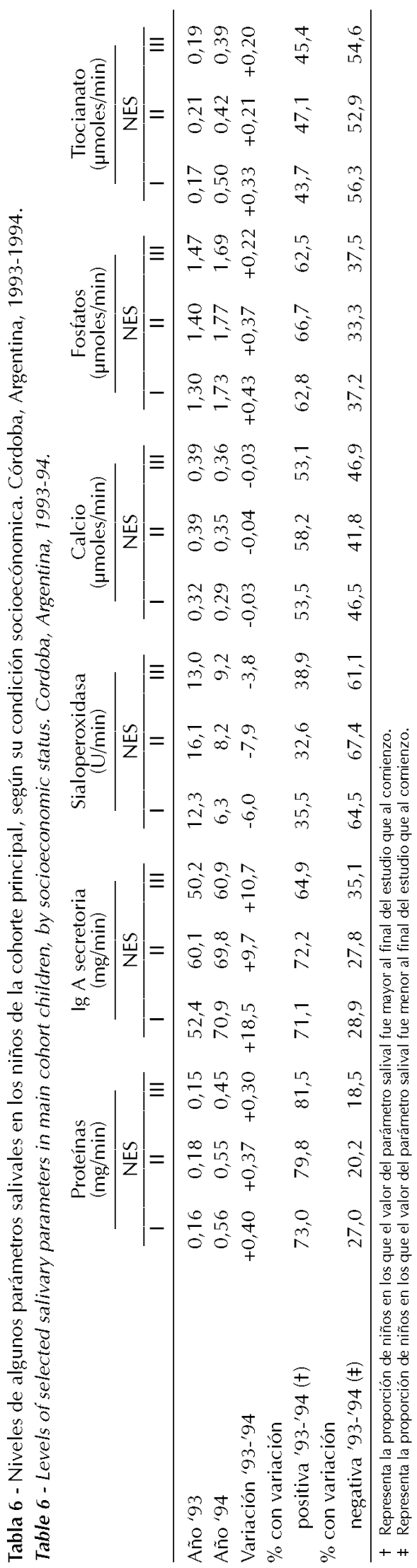


Los valores promedio de las concentraciones de proteínas totales, calcio, fosfatos, tiocianatos e Ig A secretoria y de la actividad de sialoperoxidasa al comienzo y final del estudio, las correspondientes variaciones y la proporción de niños que experimentó aumento o descenso en dichas variables entre los años '93 y '94 se indican en la Tabla 6. Aunque en algunos de esos parámetros se registraron leves diferencias interniveles, la gran dispersión de los valores individuales en cada categoría socioeconómica determinó que tales desigualdades carezcan de significación estadística. La velocidad de flujo salival (mL/minuto) al comienzo y final del estudio fueron 0,38 y 0,46 (NES I), 0,41 y 0,47 (NES II) y 0,35 y 0,42 (NES III), respectivamente.

\section{DISCU SIÓ N}

Los resultados de la presente investigación confirman observaciones previas ${ }^{7}$ acerca de la reducción de la prevalencia de caries en la dentición temporaria de la población preescolar de la Ciudad de Córdoba, en el curso de los últimos 20 años, y la existencia de fuertes desigualdades en la intensidad de la enfermedad conforme a su condición socioeconómica.

No obstante tal declinación, esta enfermedad infecciosa constituye aún un problema de salud pública para nuestra población infantil, ya que alrededor de las dos terceras partes de los niños de 4-5 años han experimentado lesiones de caries en sus dientes deciduos, la mayoría de las cuales se mantiene en actividad. Situaciones similares a la descripta fueron reportadas en varios países. En EE.UU., la declinación de caries en dentición primaria ha sido más lenta que en la dentición permanente, afectando actualmente la enfermedad a casi el $50 \%$ de la población de 5 años o menos ${ }^{30}$. En Holanda, Frencken y col. ${ }^{11}$ comunicaron que el ceo-s de niños de 6 años tuvo un descenso entre 1973 y 1988, pero advierte que entre 1982 y 1988 ha ocurrido muy poco cambio, sugiriendo una leve tendencia ascendente. Por su parte, Holt $^{15}$ señala que la prevalencia de caries disminuyó en preescolares ingleses en la década del '70, mas dicha tendencia no continuó en los años '80, en tanto que Pitts ${ }^{34}$ informa que ha cesado la caída en la frecuencia de caries en niños escoceses de 5 años, con escasa variación entre 1987 y 1991.

Diversos hallazgos avalan el criterio de que la distribución de las caries no es uniforme en toda la población. En concordancia con ello, puede se comprobar que muchos preescolares de la Ciudad de Córdoba están libres de caries; contrariamente, la afección es muy frecuente en la población infantil perteneciente a familias que padecen diversas formas de privación económica y social (NES III). Este grupo de niños presenta asimismo importantes desventajas en el estado general de salud bucodental, situación que tiende a agravarse al aumentar la edad. Todo ello permite considerar a los niños del NES III como un grupo potencialmente en riesgo de sufrir daño en el sistema estomatológico. La información disponible sugiere que esta correlación entre variables económicosociales y prevalencia de caries no representa un fenómeno excepcional. En las poblaciones infantiles hispánica, nativa y negra residente en EE.UU., los índices de caries son mucho más altos que en comunidades blancas ${ }^{45}$. También son mayores y más frecuentes los problemas periodontales y de maloclusión ${ }^{45}$ y la erosión dentaria ${ }^{28}$. Similar modelo de distribución diferencial de caries fue observado en otras partes del mundo ${ }^{12,39}$.

Como señalaron O'Sullivan y Tinanoff ${ }^{33}$, el desarrollo de las lesiones cariosas en dentición temporaria puede seguir diferentes patrones, siendo el de fosas y fisuras uno de los más frecuentes. No resulta sorprendente, entonces, que la mayor incidencia de caries registrada en la presente investigación, particularmente en los niños del nivel socioecónomico bajo, haya ocurrido en las superficies oclusales del primero y segundo molar. Similares hallazgos fueron comunicados en las poblaciones infantiles finlande$\mathrm{sa}^{17} \mathrm{y}$ norteamericana ${ }^{25}$, donde la prevalencia de caries en incisivos y caninos es muy baja. La mayor vulnerabilidad de las superficies oclusales puede obedecer a dos razones. En primer lugar, las fosas y fisuras constituyen los sitios más retentivos de las superficies dentarias, fácilmente colonizadas por bacterias cariogénicas después de la exposición del diente al ambiente bucal. En segundo término, el desplazamiento de la película salival y la depuración de azúcares se realiza más lentamente en torno a las fosas y fisuras, sobre todo de molares inferiores, por lo que la limpieza natural de estas superficies oclusales es menor ${ }^{9}$.

$\mathrm{Al}$ igual que muchas otras afecciones humanas, la caries dental es una enfermedad de etiología multifactorial. En este contexto, el consumo de azúcares ha sido considerado un factor esencial en la producción de caries ${ }^{31}$. Para muchos microorganismos bucales, y en especial aquellos con actividad cariogénica (Streptococcus mutans y Lactobacilos), dichos carbohidratos representan los principales sustratos energéticos, por cuya degradación se forman importantes cantidades de ácidos capaces de iniciar la 
desmineralización del esmalte. Además, varios mono y disacáridos son sustratos para la síntesis de polímeros extracelulares, los que al favorecer la adherencia de las bacterias a la placa dental promueven el avance de las lesiones.

Si bien diversos estudios epidemiológicos han mostrado una relación positiva entre el consumo de azúcares y la prevalencia de caries dental ${ }^{3,19}$, también hay pruebas que rechazan cualquier asociación entre dichas variables, o que la misma existe pero es muy débil ${ }^{6,38}$. Los resultados de la presente investigación muestran que, si bien los preescolares del nivel económicosocial más bajo tenían un consumo de azúcar que en cantidad y frecuencia era superior al de las restantes categorías socioeconómicas, los niños con más alto incremento de caries no consumieron cantidades significativamente mayores de azúcares, ni el consumo fue francamente más reiterado que en los demás. Tales hallazgos deben interpretarse cuidadosamente. En primer lugar, estos principios inmediatos no fueron ingeridos al estado puro, sino como componentes de alimentos con distinta cohesión, viscosidad y retentividad, características que condicionan el poder cariogénico de los mismos. En segundo lugar, la formación de polímeros extracelulares adherentes a partir de mono y disacáridos dietéticos es esencial para el desarrollo de las caries en superficies lisas ${ }^{10}$, pero no en las de fosas y fisuras ${ }^{27}$. Como en los niños de la categoría socioeconómica más baja las nuevas lesiones de caries ocurrieron predominantemente en superficies oclusales $(66,3 \%)$, se justifica que el mayor consumo de azúcares no haya influido notablemente en la incidencia de caries. La hipótesis de que la depuración bucal de azúcares podría ser mayor en los niños del NES III que en los restantes preescolares parece muy poco consistente, puesto que el volumen minuto de saliva, una de las principales variables de la cual depende dicho parámetro farmacocinético, fue muy similar en todos los niveles.

Los valores promedio inicial y final de los parámetros salivales bioquímicos (calcio, fosfatos, tiocianatos, proteínas, sialoperoxidasa) e inmunológico (IgA secretoria) analizados en la presente investigación, así como su correspondiente variación a lo largo del estudio, fueron muy similares en los tres NES. Tampoco se registraron diferencias significativas en dichas variables entre preescolares que experimentaron nuevas lesiones de caries durante el período de ensayo (TI\% > 0) y los que no sufrieron cambios en los índices de salud dental durante el mismo $(\mathrm{TI} \%=0)($ datos no presentados). El mismo hecho fue observado y analizado en el estudio trans- versal desarrollado recientemente por nosotros ${ }^{7}$. Una consideración especial merecen Ig A secretoria y sialoperoxidasa, en razón que se les ha atribuido a estas proteínas funciones específicas de defensa de la cavidad bucal frente a la agresión microbiana ${ }^{36,41}$. Aunque lo expresado anteriormente haría prever algún grado de correlación de estas proteínas salivales con la experiencia de caries, los presentes resultados indican lo contrario. A una conclusión similar arribaron Rose y $\mathrm{col}^{36}$, al demostrar que el contenido total de Ig A secretoria salival no difiere significativamente entre niños resistentes y susceptibles a la caries, si bien el contenido de anticuerpos Ig A contra Streptococcus mutans es mayor en aquéllos. En cuanto a la sialoperoxidasa, los resultados de estudios epidemiológicos son muy contradictorios, existiendo pruebas tanto a favor como en contra de una asociación con la caries dental ${ }^{41}$.

Teniendo en cuenta el carácter infecto-contagioso de la caries ${ }^{44}$, la eliminación mecánica o química de la placa dentobacteriana, o al menos la inhibición de los microorganismos patógenos que contiene, sirve a los fines de evitar o reducir la enfermedad. Entre los fármacos ensayados en humanos para el control de placa, los fluoruros constituyen uno de los grupos con mayor eficacia. Aplicados bajo la forma de tópicos en concentraciones muy altas de $\mathrm{F}^{-}$desarrollan un importante efecto cariostático, al interferir ciertos procesos enzimáticos relacionados con el metabolismo microbiano, produciendo un efecto antibacteriano directo e incrementando la formación de apatita adamantina menos susceptible a la disolución áci$\mathrm{da}^{42}$. Como resultado de ello, la aplicación local de $\mathrm{F}^{-}$altera la composición microbiana de la placa, particularmente al reducir la cantidad relativa de Streptococcus mutans ${ }^{20}$.

De la presente investigación surge que la incidencia de caries en los niños del NES III que realizaron higiene dental asistida o recibieron una o dos aplicaciones tópicas fluoruradas alcanzan tasas casi idénticas a las que tenían sus similares no tratados del NES I $(0,31,0,23$ y 0,22 vs 0,21 , respectivamente). Estos hallazgos permiten sugerir que las diferencias interniveles advertidas en el estado de salud dental de los preescolares de la Ciudad de Córdoba obedecen, al menos en parte, a las condiciones de higiene oral y/o de resistencia de las estructuras dentarias. En el estudio desarrollado por Calamari y col. ${ }^{7}$, el hábito de cepillado dental parecía no influir en los índices de caries de la población preescolar, lo cual fue atribuido a la deficiente ejecución de la limpieza mecánica por parte de los propios niños, por cuanto la eficacia del cepillado está determinada 
por factores tales como técnica empleada, duración del procedimiento y conocimiento que tienen al respecto los pacientes. Como señalaron Tsamtsouris y col. ${ }^{43}$, el asesoramiento y supervisión producen una significativa reducción en el nivel de placa dental en niños en edad preescolar.

Los resultados permiten concluir que la aplicación oportuna de medidas preventivas, como lo son

\section{REFEREN CIAS BIBLIO GRÁFICAS}

\section{BATTELLINO, L.J.; CATTONI, S.T.D. de; ESCUDERO,} M. A.; SABULSKY, J.; HIDALGO, P.H. Medidas antropométricas, salud bucodental y desarrollo psicológico en preescolares (5 años) de la Ciudad de Córdoba. Rev. Fac. Cienc. Med., (Córdoba), 32:321-42, 1974.

2. BETTS, R.H.\& DOUNTON, F.S. Electron transfer and other processes involved in the spontaneous bleaching of acidified aqueous solutions of ferric thiocyanate. Am. Chem. Soc., 75:5721-7, 1954.

3. BOWEN, W.H. \& BIRKHED, D. Dental caries: dietary and microbiology factors. In: Granath, L. \& Mc Hugh, W.D. ed. Systematized prevention of oral disease: theory and practice. Boca Raton, CRC, 1986, p. 19-41.

4. BRETZ, W.A.; DJAHJAH, C.; ALMEIDA, R.S.; HUJOEL, P.P.; LOESCHE, W. J. Relationship of microbial and salivary parameters with dental caries in Brazilian pre-school children. Community Dentistry Oral Epidemiol., 20:261-4, 1992.

5. BRONFMAN, M. \& TUIRÁN, R. La desigualdad ante la muerte: clases sociales y mortalidad. Cuad. Med. Soc., Rosario, 26(30):53-75, 1984.

6. BURT, B.A.; EKLUND, S.A.; MORGAN, K.J.; LARKIN, F.E.; GUIRE, K.E., BROWN, L.O.; WEINTRAUB, J.A. The effects of sugars intake and frequency of ingestion on dental caries increment in a three-year longitudinal study. J. Dent. Res., 67:1422-9, 1988.

7. CALAMARI, S.E.; AZCURRA, A.I.; BATTELLINO, L.J.; TRECO, G.G.F. de. Condición socieconómica, salud bucodental y factores asociados en la población infantil (5 años) de la Ciudad de Córdoba (Argentina). Rev. Dent. Chile, 86:9-17, 1995.

8. CHEN, P.S.; TORIBARA, T.Y.; WARNER, H. Microdetermination of phosphorus. Anal. Chem., 28:1756-8, 1956.

9. DAWES, C. \& MACPHERSON, L.M.D. The distribution of saliva and sucrose around the mouth during the use of chewing gum and the implications for the site specificity of caries and calculus deposition. J. Dent. Res., 72:852-7, 1993.

10. FIRESTONE, A.R. \& MUHLEMANN, H.R. In vivo $\mathrm{pH}$ of plaque-covered and plaque-free interdental surfaces in humans following a sucrose rinse. Clin. Prev. Dent., 7:24-6, 1985.

11. FRENCHEN, J.E.; KALSBEECK, H.; VERRIPS, G.H. Has the decline in dental caries been healted? Changes in caries prevalence amongst 6- and 12-year-old children in Friesland, 1973-1988. Int. Dent. J., 40:225-30, 1990. la educación para reducir la ingesta de azúcares, el cepillado dental asistido y las aplicaciones tópicas fluoradas, pueden resultar muy eficaces en el control de caries en la población infantil, especialmente en aquellos grupos de niños que están social y económicamente desfavorecidos, que son precisamente donde se concentran las afecciones bucodentales.

12. GRATRIZ, D. \& HOLLOWAY, P.J. Factors in deprivation associated with dental caries in young children. Comm. Dentistry Health, 11:66-70, 1994.

13. GREENE, J.C. \& VERMILLION, J.R. The simplified oral hygiene index. J. Am. Dent. Assoc., 68:7-13, 1964.

14. GRUEBBEL, A.O. A measurement of dental caries prevalence and treatment service for deciduous teeth. $J$. Dent. Res., 23:163-8, 1944.

15. HOLT, R.D. Caries in preschool child: British trends. $J$. Dentistry, 18:296-9, 1990.

16. KALSBEEK, H. \& VERRIPS, G.H.W. Dental caries prevalence and the use of fluorides in European countries. $J$. Dent. Res., 69(Spp Iss):728-32, 1990.

17. KEROSUO, H. \& HONKALA, E. Caries experience in the primary dentition among groups of Tanzanian and Finnish 3to 7-year-old children. Comm. Dentistry Oral Epidemiol., 19:272-6, 1991.

18. KOCH, A.L.; GERSHEN, J.A.; MARCUS, A. A children's oral status index based on dentist judgment. J. Am. Dent. Assoc., 110:36-42, 1985.

19. LACHAPPELLE-HARVEY, D. \& SEVIGNY, J. Multiple regression analysis of dental status and related food behaviour of French Canadian adolescents. Comm. Dentistry Oral Epidemiol., 13:226-9, 1985.

20. LOESCHE, W.J.; SYED, S.A.; MURRAY, R.J.; MELLBERG, J.R. Effect of topical acidulated phosphate fluoride on percentage of Streptococcus mutans and Streptococcus sanguis in plaque. II. Pooled occlusal and pooled approximal samples. Caries Res., 9:139-55, 1975.

21. LOMBARDI, C.; BRONFMAN, M.; FACHINI, C.; VICTORA, C.G.; BARROS, F. Operacionalização do conceito de classe social em estudos epidemiológicos. Rev. Saúde Pública, 22:253-65, 1988.

22. LOWRY, O.H.; ROSENBROUGH, N.J.; FARR, A.L.; RANDALL, R.J. Protein measurement with the Folin phenol reagent. J. Biol. Chem., 193:265-75, 1951.

23. MANCINI, G.; CARBONARA, A.O. HEREMANS, J.F. Immunochemical quantitation of antigens by single radial immunodiffusion. Immunochemistry, 2:235-7, 1965.

24. MANJI, F. \& FEJERSKOV, O. Dental caries in development countries in relation to the appropriate use of fluoride. $J$. Dent. Res., 69(Sp Iss):733-41, 1990. 
25. MARGOLIS, M.Q.; VANN, W.F.; HUNT, R.J.; STEWART, P.W. Distribution of primary tooth caries in first-grade children from nonfluorinated US communities. Pediatr. Dentistry, 16:200-5, 1994.

26. MARTHALER, T.M. Caries status in Europe and predictions of future trends. Caries Res., 24:381-96, 1990.

27. MCDONALD, J.L. \& STOOKEY, G.K. Animal studies concerning the cariogenicity of dry breakfast cereals. $J$. Dent. Res., 56:1001-6, 1977.

28. MILLWARD, A.; SHAW, L.; SMITH, A. Dental erosion in four-year-old children from differing socioeconomic backgrounds. J. Dent.Child., 61:263-6, 1994.

29. NAKANO, K. \& ASADA, K. Hydrogen peroxide is scavenged by ascorbate-specific peroxidase in spinach chloroplasts. Plant. Cell Physiol., 22:867-80, 1981.

30. NATIONAL INSTITUTE OF DENTAL RESEARCH. Epidemiology and Oral Disease Prevention Program. Oral health of United States children, the national survey of dental caries in U.S. school children: 1986-1987, 1989. (NIH Pub. No 89-2247).

31. NEWBRUN, E. Sucrose on the dynamics of carious process. Int. Dent. J., 2:13-23, 1982.

32. NIE, N.N.; HULL, H.C.; JENKINS, J.G.; STEINBRENNER, K.; BENT, D.H. Statistical package for the social sciences. 2nd ed. New York, Mc Graw Hill, 1975.

33. O'SULLIVAN, D.M. \& TINANOFF, N. Social and biological factors contributing to caries of the maxillary anterior teeth. Pediatr. Dent., 5:41-4, 1993.

34. PITTS, N.B. Recent trends in caries prevalence among 5year-olds in Scotland. Caries Res., 28:210, 1994.

35. RAY SARKAR, B.C. \& CHAUHAN, V.P.S. A new method for determinating microquantities of calcium in biological materials. Anal. Biochem., 20:155-66, 1967.

36. ROSE, P.A.; GREGORY, R.L.; GFELL, L.E.; HUGHES, C.V. Ig A antibodies to Streptococcus mutans in cariesresistant and -susceptible children. Pediatr. Dent., 16:272-5, 1994.
37. RUGG-GUNN, A.J.; HACKETT, A.F.; APPLETON, D.R.; JENKINS, G.N.; EASTOE, J.E. Relationship between dietary habits and caries increment assessed over two years in 405 English school children. Arch. Oral Biol., 29:983-92, 1984.

38. RUGG-GUNN, A.J. Diet and dental caries. In: Murray, J. J. ed. The prevention of dental disease. Oxford University Press, 1983. p 3-82.

39. SPENCER, N.; WRIGHT, F.A.C; BROWN, L.M.; BROWN, L.P. Changing caries experience and risk factors in five- and six-year-old Melbourne children. Aust. Dent. J., 34:160-5, 1989.

40. TEN CATE, J.M. \& MARSH, P.D. Procedures for establishing efficacy of antimicrobial agents for chemotherapeutic caries prevention. J. Dent. Res., 73:695-703, 1994.

41. TENOVUO, J.; GRAHM, E.; LEHTONEN, O.O.; HYYPA, T.; KASHUVAARA, L.; VILJA, P. Antimicrobial factors in saliva: ontogeny and relation to oral health. J. Dent. Res., 66:475-9, 1987.

42. THYLSTRUP, A. \& FEJERSKOV, O. Efecto del fluoruro sobre la placa dental, la estructura del diente y la caries dental. In: Caries. Fejerskov, O. ed. Buenos Aires, Edic Doyma S.A, 1988. p. 254-85.

43. TSAMTSOURIS, A.; WHITE, G.E.; CLARK, E.R. The effect of instruction and supervised toothbrushing on the reduction of dental plaque in kindergarten children. J. Dent. Child., 46:204-9, 1979.

44. VAN HOUTE, J. Role of micro-organisms in caries etiology. J. Dent. Res., 73:672-81, 1994.

45. WALDMAN, B. Paediatric dentistry in the United States: there is a long way to go. Int. J. Paediatr. Dentistry, 4:277-9, 1994.

46. WORLD HEALTH ORGANIZATION. Oral health surveys: basic methods. 3rd rev. ed. Geneva, 1987.

47. YANKILEVICH, E.R.L.M. de; CATTONI, S.T.D. de; CORNEJO, L.S.; BATTELLINO L.J. Distribución de la caries dental en niños preescolares en una región urbana, Argentina, 1992. Rev. Saúde Pública, 27:436-44, 1993. 